\title{
REMOVING TOPOGRAPHY INFLUENCE IN VLF-EM DATA BY LINEAR FILTERING
}

\author{
Niels Anders Edsen ${ }^{1}$ and Bo Holm Jacobsen ${ }^{2}$ \\ ${ }^{1}$ Laboratory of Geophysics, Department of Earth Sciences, University of Aarhus, Denmark. \\ Presently at the Danish Geotechnical Institute, Maglebjergvej 1, DK-2800 Lyngby. \\ Email: anders.edsen@geoteknisk.dk. \\ ${ }^{2}$ Laboratory of Geophysics, Department of Earth Sciences, University of Aarhus, Denmark. \\ Email: geofbhj@aau.dk
}

\begin{abstract}
When surveying upon a level homogenous halfspace or over horizontally layered lithologies no anomalies are seen in the conventional VLF-EM method. But in situations where the topography represents a surface with varying relief even upon a homogenous halfspace the terrain itself will generate VLF-EM anomalies which can be misinterpreted as originating from subsurface conductors. VLF-EM topography response is seen in the real part as well as in the ellipticity measurements. Based on a Wiener filtering concept a method is presented by which a filter can be designed and the topography effect from varying topography in Epolarization removed by simply applying this filter to the topography data. The major advantages within the suggested method are the simplicity in use and a considerable reduction in computing time compared to a numerical integration approach.
\end{abstract}

\section{INTROCUCTION}

When the strike of the topographic structures is perpendicular to the direction of wave propagation (H-polarization) the magnetic field will still be horizontal in the absence of conductive structures and no VLF-EM anomaly due to topography will occur. However, where the direction of propagation of the primary VLF-EM field follows the strike of the topography (E-polarization) the primary magnetic field will be aligned parallel to the slope and thus, measurements will be affected. Typically, the target conductors share strike direction with the general geological setting including the topography. The actual topography effect is a function of terrain relief as well as skindepth and therefore a reliable correction requires knowledge of the terrain resistivity.

The topography effect has been known for several years and it has been tradition simply to plot the topography data along the surveyed profile either as raw topography or as calculated slope in dip angle or percent. Qualitatively, a ridge will induce a false normal polarity anomaly and a valley bottom a false reversed pattern. A number of papers have tried to address this problem in a more quantitative sense. Based on model experiments and calculations on reflection of EM waves Baker and Myers (1980) and Eppelbaum (1991) respectively suggested very simple corrections only dependent on the slope of the topography at the actual measurement position. Thus, on a level position just beside a steep hill these corrections are zero disregarding the dimension of the topography feature and the terrain 
resistivity. Karous (1979) chose an analytical integral approach to the problem and found an expression for the topography effect which includes the terrain resistivity and extends the correction to include the area in the vicinity of a topographic feature. However, Karous assumed a constant phase of the EM-field at increasing depth and erroneously the correction suggested is identical for real part and ellipticity measurements.

\section{THE FILTER DEVELOPMENT}

The aim is the development of two filters for real part and ellipticity data respectively which when applied to topography data will produce the VLF-EM topography response and thus, the correction to be applied to the measured VLF-EM profile data. To simplify the problem the filter is applied to topography slopes and the basic concept can be stated in the following equation:

$$
\Delta t=a * f+s
$$

Here $a$ represents the sequence of topography slope data along the profile, $f$ denotes the filter for either real part or ellipticity, $s$ is a noise component which incorporates also the nonlinearities in the forward problem and $\Delta t$ represents the VLF-EM topography response. First we estimate the filters by a traditional Wiener filtering approach. The least-square solution (LSQ) to eq. 1 in the Fourier domain is given as $F_{L S O}=\left(A^{H} A\right)^{-1} A^{H} \Delta T$, where $H$ denotes the Hermetian transpose $\left(A^{\mathrm{H}}=\left(A^{*}\right)^{\mathrm{T}}\right)$. For a suite of synthetic topographies a robust mean solution is found as the ratio between the cross power spectrum and the auto power spectrum :

$$
\overline{F_{L S Q}}=\frac{\sum_{j=1}^{N} A_{j}{ }^{H} \Delta T_{j}}{\sum_{j=1}^{N} A_{j}{ }^{H} A_{j}} ; \quad \overline{f_{L S Q}}=\mathscr{F}^{-1}\left(\overline{F_{L S Q}}\right)
$$

The VLF-EM topography response was calculated by a simple 2D numerical integration technique. The filter obtained was seen to be of symmetric shape with a halfwidth directly proportional to the skindepth whereas the amplitude of the filter was found to be inversely proportional to the skindepth and directly proportional to the sampling distance. The general expression of the filters are given below:

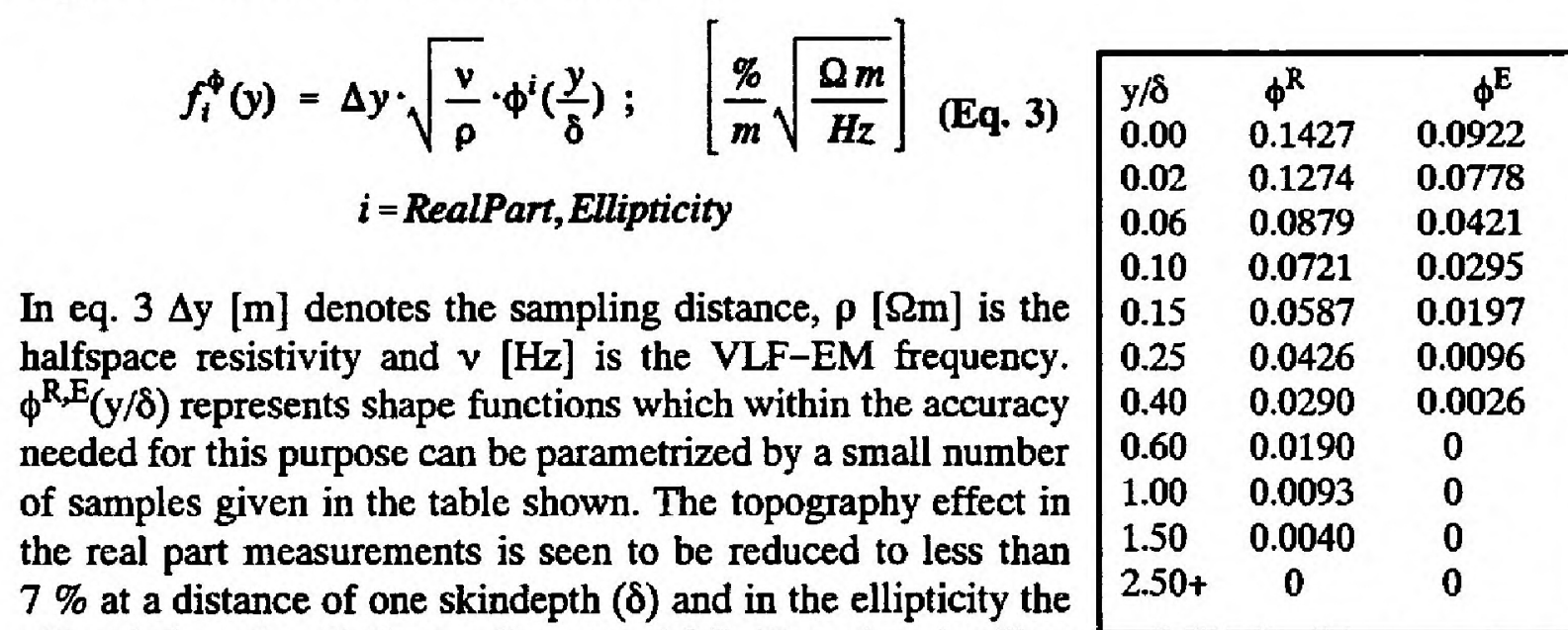
$7 \%$ at a distance of one skindepth $(\delta)$ and in the ellipticity the effect is less than $3 \%$ at a distance of $\delta / 2$. Note that the filter width is given in fractions of skindepth and before applying the filters to profile slope data the samples of the filter shape

The positive part of the symmetric shape function $\phi^{R, E}(y / \delta)$. 


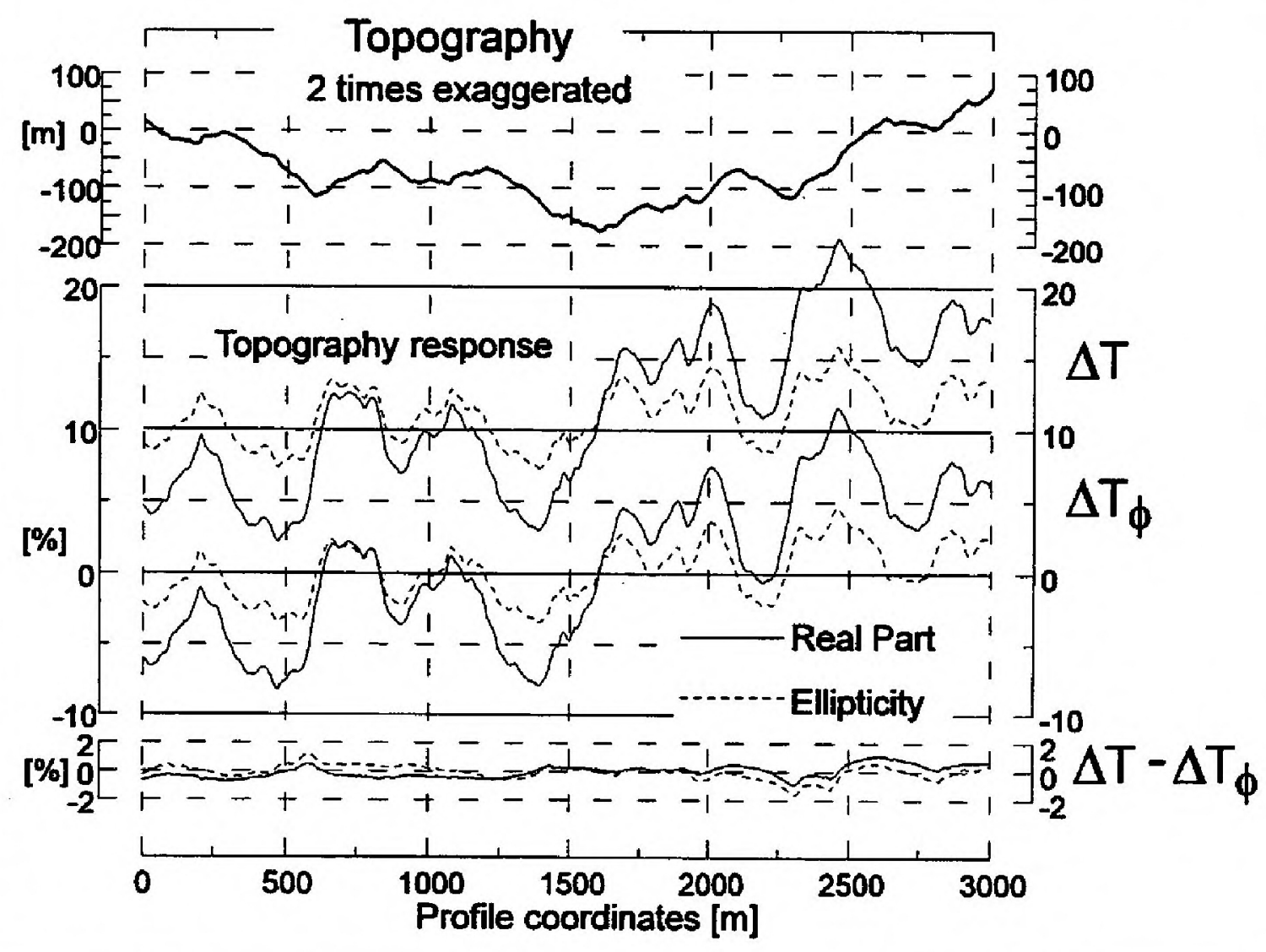

Figure 1. Numerical calculation of the VLF topography response $\Delta T(+10)$ compared to the result of linear filtering $\Delta T_{\phi}$ of the topography shown $(\rho=6400 \Omega \mathrm{m}, \Delta y=10 \mathrm{~m})$.

functions $\phi$ must be splined to the actual sampling distance in terms of skindepth used in the survey. Further details of the filter development and analysis of filter performance are given by Edsen (1997). In fig. 1 the filters of eq. 3 are applied to the slopes of the shown profile topography data. The result of the filtering are compared to the VLF-EM topography response calculated by numerical integration and the observed differences are seen to be insignificant.

\section{TOPOGRAPHY CORRECTION OF VLF-EM DATA}

The example in figure 2 is a profile from a large VLF-EM ground survey i SW Greenland by Nunaoil AVS utilizing the NAA transmitter in Cutler, Maine $(24.0 \mathrm{kHz}$ ) (Edsen, 1997). All surveyed profiles were corrected for topography influence using the method presented in this paper and the data were subsequently interpreted using the $2 \mathrm{D}$ forward modelling program by Edsen and Nissen (1997). In the example shown the condition of E-polarization of the VLFEM field is fulfilled better than 10 degrees and the halfspace resistivity was estimated from IP and resistivity measurements. Before applying the filters of eq. 3 the samples of the shape functions $\phi^{\mathrm{R}, \mathrm{E}}$ given in the table above were splined to the actual station interval of $12.5 \mathrm{~m}$ which equals $0.05 \delta$ at a halfspace resistivity of $5000 \Omega \mathrm{m}$. Borehole data were available at some of the measured profiles which enabled an extra control of the modelling performed. Only when correction of topography influence was applied it was possible to obtain a satisfactory agreement with the models expected from the drillings data. 


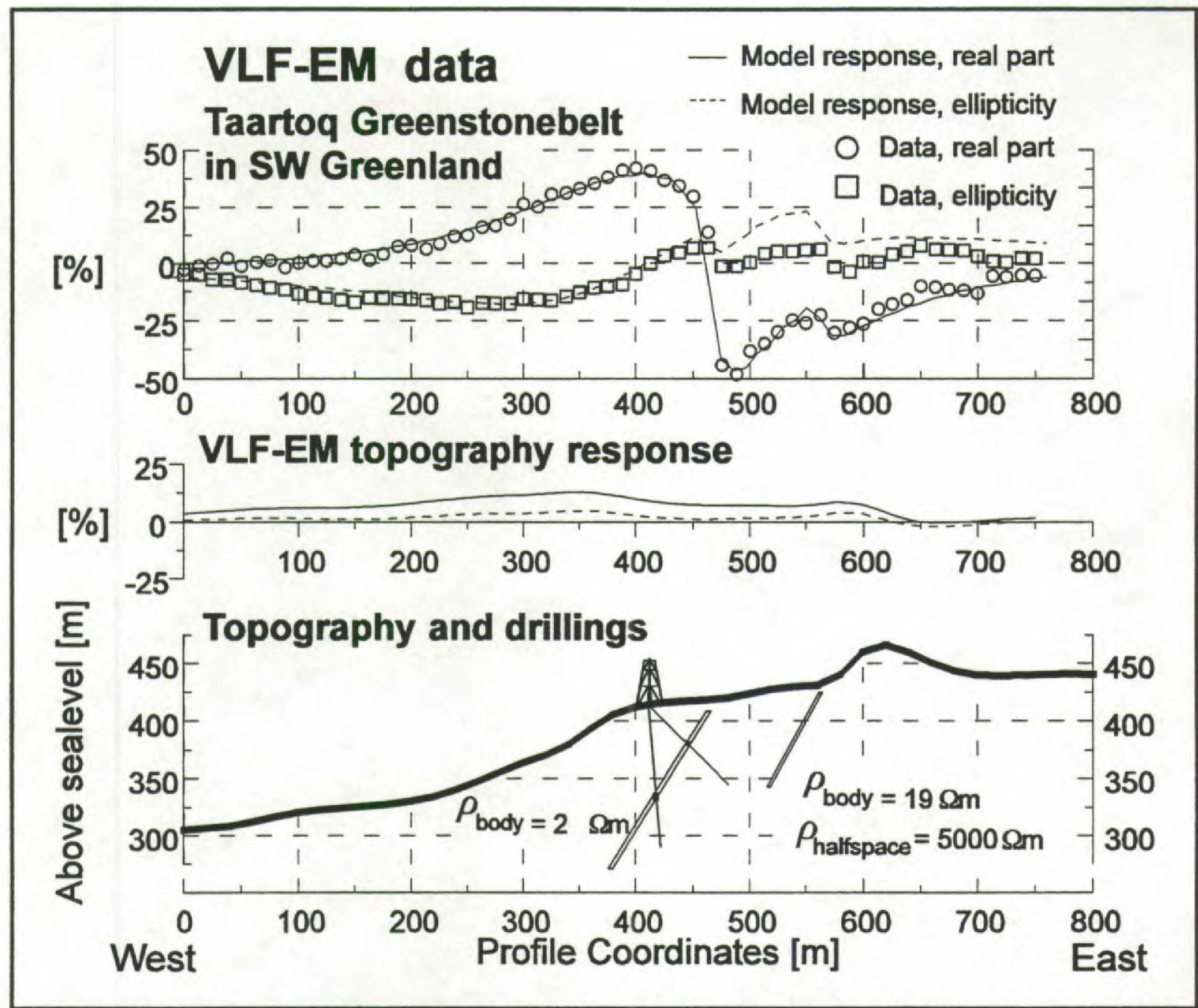

Figure 2. Modelling of VLF-EM data corrected for topography influence. VLF-EM measurements were performed with the Geonics EM-16 instrument facing East.

\section{CONCLUSION}

Through the method presented it is possible to design a simple scalable filter which produc es the VLF-EM topography correction by linear filtering of the slopes along the profile. The difference between the reference $2 \mathrm{D}$ numerical integration calculation and the correction obtained by linear filtering is seen to be insignificant and the computing time is reduced by a factor of $10^{3}$.

\section{REFERENCES}

Baker, H.A. and Myers, J.O., 1979. A topographic correction for VLF-EM profiles based on model studies. Geoexploration, 18, 135-144.

Eberle, D., 1981. A method of reducing terrain relief effects from VLF-EM data. Geoexploration, 19, 103-114.

Edsen, N.A., 1997. Geofysik og mineraleksploration; VLF metoden og dens anvendelse som en integreret del af et mineraleksplorationsprogram i Taartoq Grønstensbæltet i sydvest Grønland. MSc thesis in the danish language at the Laboratory of Geophysics, Department of Earth Sciences, University of Aarhus, Denmark. 103 pp.

Edsen, N.A. and Nissen, J., 1997. VLFMOD, a free forward VLF modelling software package. The program can be downloaded at the homepage of ABEM AB (http://www.abem.se).

Karous, M.R., 1979. Effect of relief in EM methods with a very distant source. Geoexploration, 17, 33-42. 